\title{
The Intermolecular NOE Depends on Isotope Selection: Short Range vs Long Range Behavior
}

\author{
Philipp Honegger, ${ }^{\perp}$ Maria Enrica Di Pietro, ${ }^{\perp}$ Franca Castiglione, ${ }^{*}$ Chiara Vaccarini, Alea Quant, \\ Othmar Steinhauser, Christian Schröder,* and Andrea Mele
}

Cite This: J. Phys. Chem. Lett. 2021, 12, 8658-8663

Read Online

ABSTRACT: The nuclear Overhauser effect (NOE) is a powerful tool in molecular structure elucidation, combining the subtle chemical shift of NMR and three-dimensional information independent of chemical connectivity. Its usage for intermolecular studies, however, is fundamentally limited by an unspecific longranged interaction behavior. This joint experimental and computational work shows that proper selection of interacting isotopes can overcome these limitations: Isotopes with strongly differing gyromagnetic ratios give rise to short-ranged intermolecular NOEs. In this light, existing NOE experiments need to be reevaluated and future ones can be designed accordingly. Thus, a new chapter on intermolecular structure elucidation is opened.

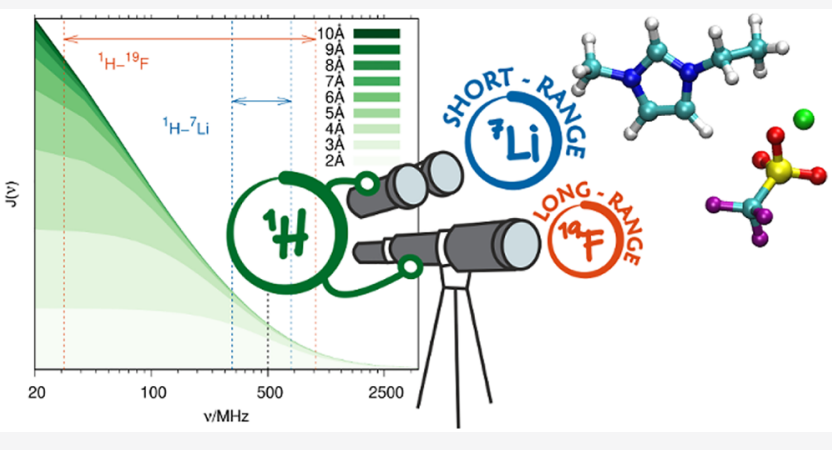

$$
G(t)=\left\langle\frac{1}{r(0)^{3}} \frac{1}{r(t)^{3}}\left(\frac{3}{2} \cos ^{2}(\theta(t))-\frac{1}{2}\right)\right\rangle
$$

with $\vec{r}(t)$ as the vector connecting the two interacting nuclei $I$ and $S$ at time $t$, and $\theta(t)$ is the angle swept by this vector during timespan $t$. In the frequency domain, this corresponds to the spectral density function (SDF)

$$
J(\nu)=\operatorname{Re}\left[\int_{0}^{\infty} \mathrm{e}^{-i 2 \pi \nu t} G(t) \mathrm{d} t\right]=\int_{0}^{\infty} \cos (-2 \pi \nu t) G(t) \mathrm{d} t
$$

information on molecular structure and processes. Since the interpretation of such spectral features is often nontrivial and may lead to contradicting viewpoints, it can be augmented by molecular dynamics (MD) simulations which serve as a mathematical microscope into the atomistic world. ${ }^{4-6}$

The tremendous synergy of spectroscopic methods and MD simulations is the charming toolkit for understanding interactions in liquid media leading to a particular structure and corresponding physicochemical properties. In 1995, the pioneering NMR paper by Osteryoung first showed the potential of the nuclear Overhauser effect (NOE) as a detection tool of intermolecular contacts in liquids that opens the route to NOE-based investigations on the local structure of ILs. ${ }^{8}$ The unique role of NOE in the large repertoire of NMR techniques is related to the fact that NOE depends on spatial dipolar interactions of nuclei rather than chemical connectivity via chemical bonds. Consequently, NOE is a powerful tool to characterize the structure, interaction, and dynamics in liquids. ${ }^{9,10}$ On the level of molecular processes, the temporal evolution (randomization rate) of the NOE is described by the time correlation function (TCF) with Re extracting the real part of the Fourier transformation. Cross-relaxation rates are then calculated using Larmor frequencies $\nu_{I}=\gamma_{I} \nu$ and $\nu_{S}=\gamma_{S} \nu$ :

$$
\sigma_{\mathrm{L}}(\nu)=0.6 J\left(\nu_{I}+\nu_{S}\right)-0.1 J\left(\nu_{I}-\nu_{S} \mid\right)
$$

The intramolecular NOE has become a standard technique in molecular structure elucidation. The distance of the interacting nuclei $\vec{r}$ is constant except for molecular vibrations and segmental motion and depends only on molecular rotation. This yields a strict $1 / r^{6}$ distance dependence that ensures that a meaningful NOE can only occur up to $4-5 \AA^{11}$ This wellknown distance dependence mediated by dipole-coupling can

Received: July 13, 2021

Accepted: August 16, 2021

Published: September 2, 2021 
be understood from eq 1 . The situation changes for intermolecular NOEs, where the interacting spins are not located on the same molecule: ${ }^{12}$

- A reference spin interacts with many surrounding spins. Instead of one internuclear distance, there is a distribution of distances, known as the radial distribution function (RDF). We are primarily interested in the contact shell surrounding a reference molecule. The molecules beyond form the bulk (Figure S1). The number of partner spins increases by an order of $r^{2}$ with increasing distance.

- The greater the distance between two interacting spins, the more time the spin-joining vector needs to randomize its length and orientation. The randomization time also increases by order of $r^{2}$ (Figure S2).

- Summing up over all spherical distance shells $r$ adds an order of $r$.

Thus, in the extreme case, using model theory, Halle predicted a long-ranged intermolecular NOE decaying by an order of 1 / $r{ }^{12} \mathrm{MD}$ simulations expanding on those models showed a somewhat more beneficial but still long-ranged behavior between $1 / r$ and $1 / r^{3} .{ }^{13}$ Structural short-ranged information is present in intermolecular NOEs too but buried by unspecific magnetization transfers between the reference molecule and a multitude of distant bulk molecules. ${ }^{14}$

The lower the frequency of the spectral density function $J(\nu)$, the more long-ranged the NOE becomes. As pointed out by Weingärtner and co-workers in their seminal paper, the interaction range depends on the spectrometer frequency. ${ }^{15}$

The approach proposed by Castiglione et al. in ref 16 and treated in this work seeks to select general cases in which the short-distance based interpretation on NOE still holds but, at the same time, does not contradict the general theory of intermolecular cross-relaxation. The cross-relaxation rate $\sigma_{\mathrm{L}}$ is a linear combination of the high-frequency part $J\left(\nu_{I}+\nu_{S}\right)$ and the low-frequency part $J\left(\left|\nu_{I}-\nu_{S}\right|\right)$ of the SDF. Indeed, the long-ranged contributions of $J(\nu \rightarrow 0)$ can be made negligible by selecting isotopes maximizing the frequency difference $\nu_{I}-$ $\nu_{S} \mathrm{I}=\mathrm{I}\left(\gamma_{I}-\gamma_{S}\right) \cdot \nu \mathrm{l}$.

In this instance, we propose that the heteronuclear NOE of ${ }^{1} \mathrm{H}\left(\gamma_{\mathrm{H}}=42.577 \mathrm{MHz} \mathrm{T}{ }^{-1}\right)$ and ${ }^{7} \mathrm{Li}\left(\gamma_{\mathrm{Li}}=16.546 \mathrm{MHz} \mathrm{T}{ }^{-1}\right)$ is of a shorter range than the one between ${ }^{1} \mathrm{H}$ and ${ }^{19} \mathrm{~F}\left(\gamma_{\mathrm{F}}=\right.$ $\left.40.078 \mathrm{MHz} \mathrm{T}{ }^{-1}\right)$. This theory was tested by O'Dell and coworkers using a combination of MD simulations and quantitative HOESY analysis. ${ }^{17,18}$ The elegant (yet not straightforward) fit of suitably normalized HOESY build-up curves with a modified expression including both the longitudinal relaxation times and diffusion coefficients allows for the precise calculation of absolute intermolecular crossrelaxation rates and their comparative use between different ionic liquids, concentrations, or temperatures. ${ }^{19,20}$

The following calculations are based on a $500 \mathrm{MHz}{ }^{1} \mathrm{H}$ NMR spectrometer (cf. eq 3):

- In the homonuclear extreme case, $J(2 \nu)$ and $J(0)$ contribute, e.g., $2 \nu=1000 \mathrm{MHz}$ for ${ }^{1} \mathrm{H}$. The highfrequency part is short-ranged but the low-frequency part is long-ranged, as can be seen in the top panel of Figure 1, rendering the ${ }^{1} \mathrm{H}-{ }^{1} \mathrm{H}$ NOEs long-ranged.

- The same is true for ${ }^{1} \mathrm{H}-{ }^{19} \mathrm{~F}$ NOEs. The gyromagnetic ratios are similar $\left(\nu_{I}+\nu_{S}=970 \mathrm{MHz}\right.$ and $\left|\nu_{I}-\nu_{S}\right|=30$ $\mathrm{MHz}$ ) resulting in similar problems as the homonuclear case.
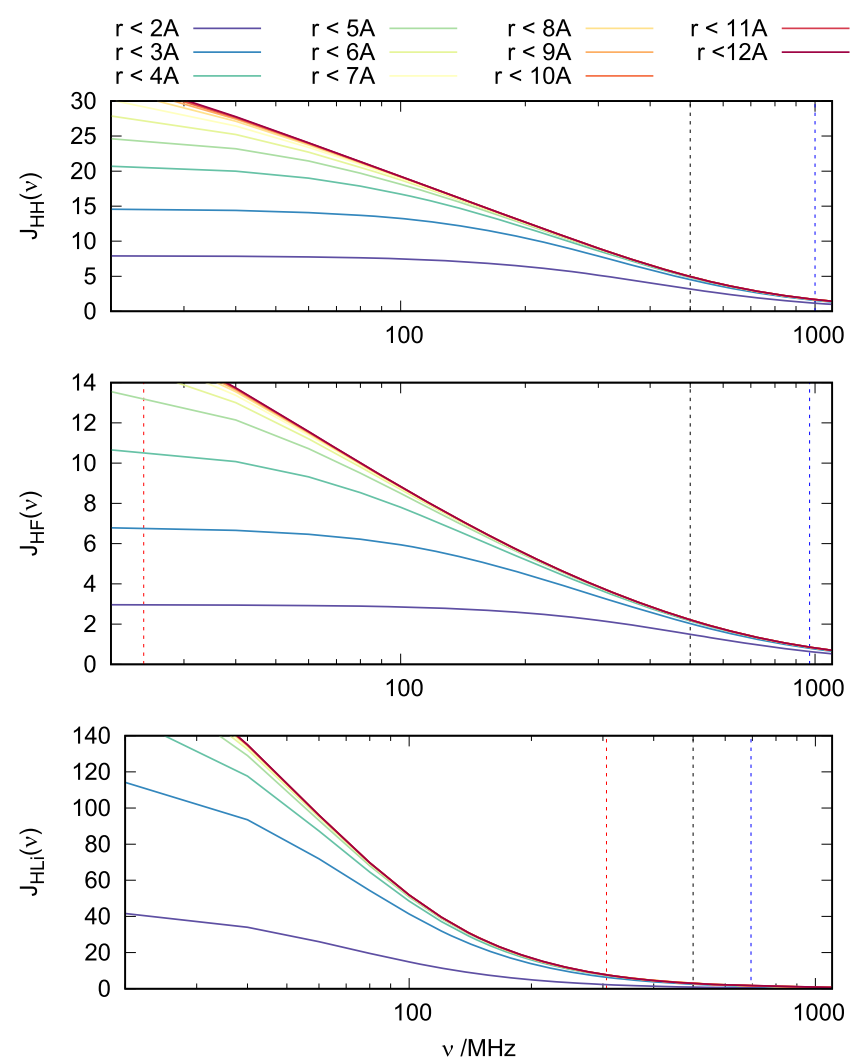

Figure 1. SDF $J(\nu)$ of the H8-H8 (top), H8-F (middle), and H8$\mathrm{Li}$ (bottom) spin pairs, resolved into cumulative contributions. The lower the frequency, the more long-ranged the $J(\nu)$ becomes. Explicitly marked frequencies are the following: spectrometer (black), high-frequency contribution $J\left(\nu_{I}+\nu_{S}\right)$ (blue), low-frequency contribution $J\left(\nu_{I}-\nu_{S}\right)$ (red, beyond range for ${ }^{1} \mathrm{H}-{ }^{1} \mathrm{H}$ )

- For ${ }^{1} \mathrm{H}-{ }^{7} \mathrm{Li} \mathrm{NMR}$, the gyromagnetic ratios differ significantly; hence both terms at $\nu_{I}+\nu_{S}=694 \mathrm{MHz}$ and $\left|\nu_{I}-\nu_{S}\right|=306 \mathrm{MHz}$ draw from a similar part of the SDF and avoid the low-frequency part, prospecting a more beneficial short-ranged behavior.

The less similar the gyromagnetic ratios, the higher is the lowfrequency $\left|\nu_{I}-\nu_{S}\right|$, avoiding the long-ranged low-frequency SDF limit $J(\nu \rightarrow 0)$.

In this proof-of-concept study, we perform semiquantitative experimental NOE measurements of the ionic liquid/salt solution 0.9 1-ethyl-3-methylimidazolium $\left[\mathrm{C}_{2} \mathrm{MIm}\right] \cdot 0.1 \mathrm{Li} \cdot 1.0$ triflate [OTf] (Scheme 1). The simple fit of the HOESY build-

Scheme 1. Chemical Structure of the IL/Salt Mixture

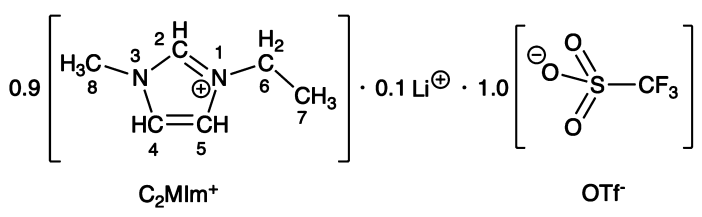

up curves with the fundamental expression derived by Solomon equations ${ }^{11,21}$ gives relative cross-relaxation rates, with no need for elaborate normalization or fitting procedures. Still, we demonstrate that these relative values do reflect the heteronuclear proximity when interpreted bearing in mind the isotope dependency of short- and long-range contributions. This joint theoretical and experimental validation represents 
$\stackrel{{ }^{1} \mathrm{H}-{ }^{-19} \mathrm{~F}}{\left[\mathrm{C}_{2} \mathrm{MIm}\right][\mathrm{OTf}]}$
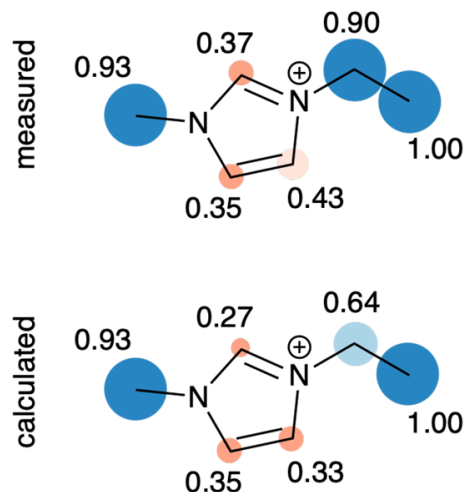

experiment computation

$\left[\mathrm{C}_{2} \mathrm{Mlm}\right][\mathrm{OTf}]$

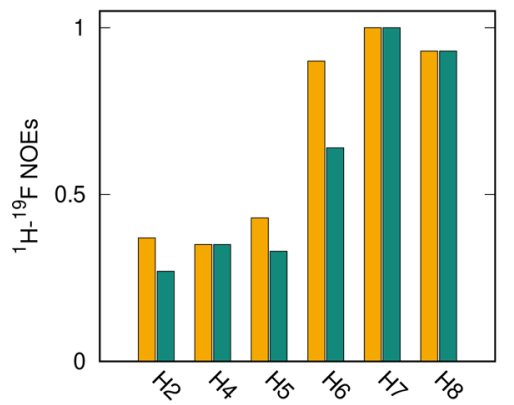

${ }^{1} \mathrm{H}-{ }^{19} \mathrm{~F}$

$0.9\left[\mathrm{C}_{2} \mathrm{MIm}\right] 0.1[\mathrm{Li}] 1.0[\mathrm{OTf}]$

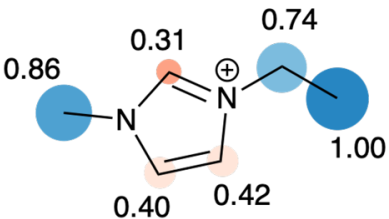

0.72

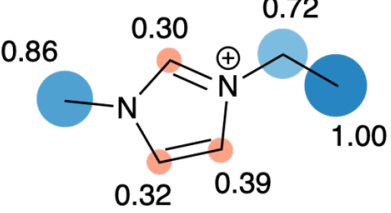

$0.9\left[\mathrm{C}_{2} \mathrm{MIm}\right] 0.1[\mathrm{Li}] 1.0[\mathrm{OTf}]$

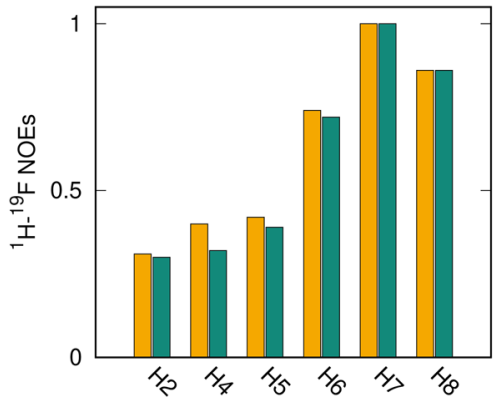

${ }^{1} \mathrm{H}-{ }^{7} \mathrm{Li}$

$0.9\left[\mathrm{C}_{2} \mathrm{MIm}\right] 0.1[\mathrm{Li}] 1.0[\mathrm{OTf}]$
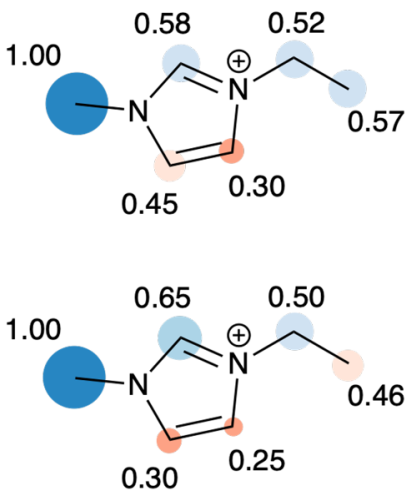

$0.9\left[\mathrm{C}_{2} \mathrm{MIm}\right] 0.1[\mathrm{Li}] 1.0[\mathrm{OTf}]$

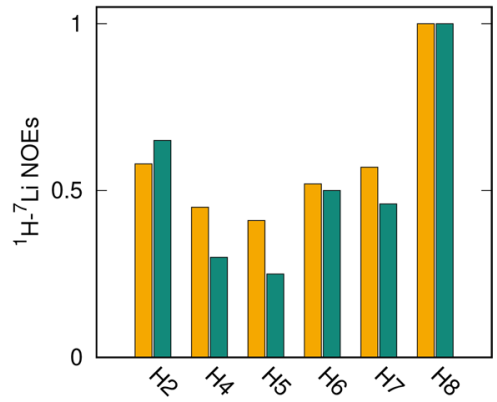

Figure 2. Top: Experimental (upper row) and computational (bottom row) $\mathrm{C}_{2} \mathrm{MIm}^{+}-\mathrm{F}$ and $\mathrm{C}_{2} \mathrm{MIm}^{+}-\mathrm{Li}$ NOEs for the neat IL $\left[\mathrm{C}_{2} \mathrm{MIm}\right][\mathrm{OTf}]$ and its mixture with $[\mathrm{Li}][\mathrm{OTf}]$, normalized to the most intense NOE signal per molecule. Experimental parameters: $T=298 \mathrm{~K}$, mixing times were 900 $\mathrm{ms}\left(\mathrm{C}_{2} \mathrm{MIm}^{+}-\mathrm{F}\right)$ and $1200 \mathrm{~ms}\left(\mathrm{C}_{2} \mathrm{MIm}^{+}-\mathrm{Li}\right)$. Bottom: Comparison of experimental and simulated NOEs.

a)

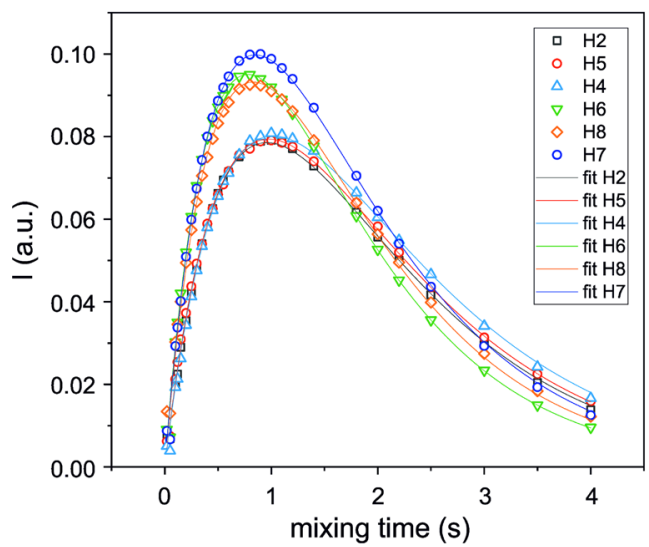

b)

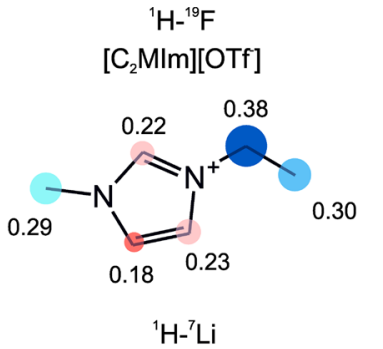

$0.9\left[\mathrm{C}_{2} \mathrm{MIm}\right]$ 0.1[Li'] 1.0[OTf]

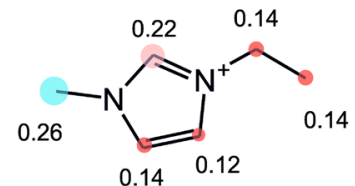

${ }^{1} \mathrm{H}-{ }^{19} \mathrm{~F}$

$0.9\left[\mathrm{C}_{2} \mathrm{MIm}\right]$ 0.1[Li+] 1.0[OTf]

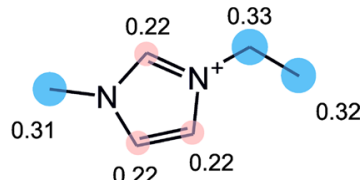

$0.22 \quad 0.22$

\section{2}



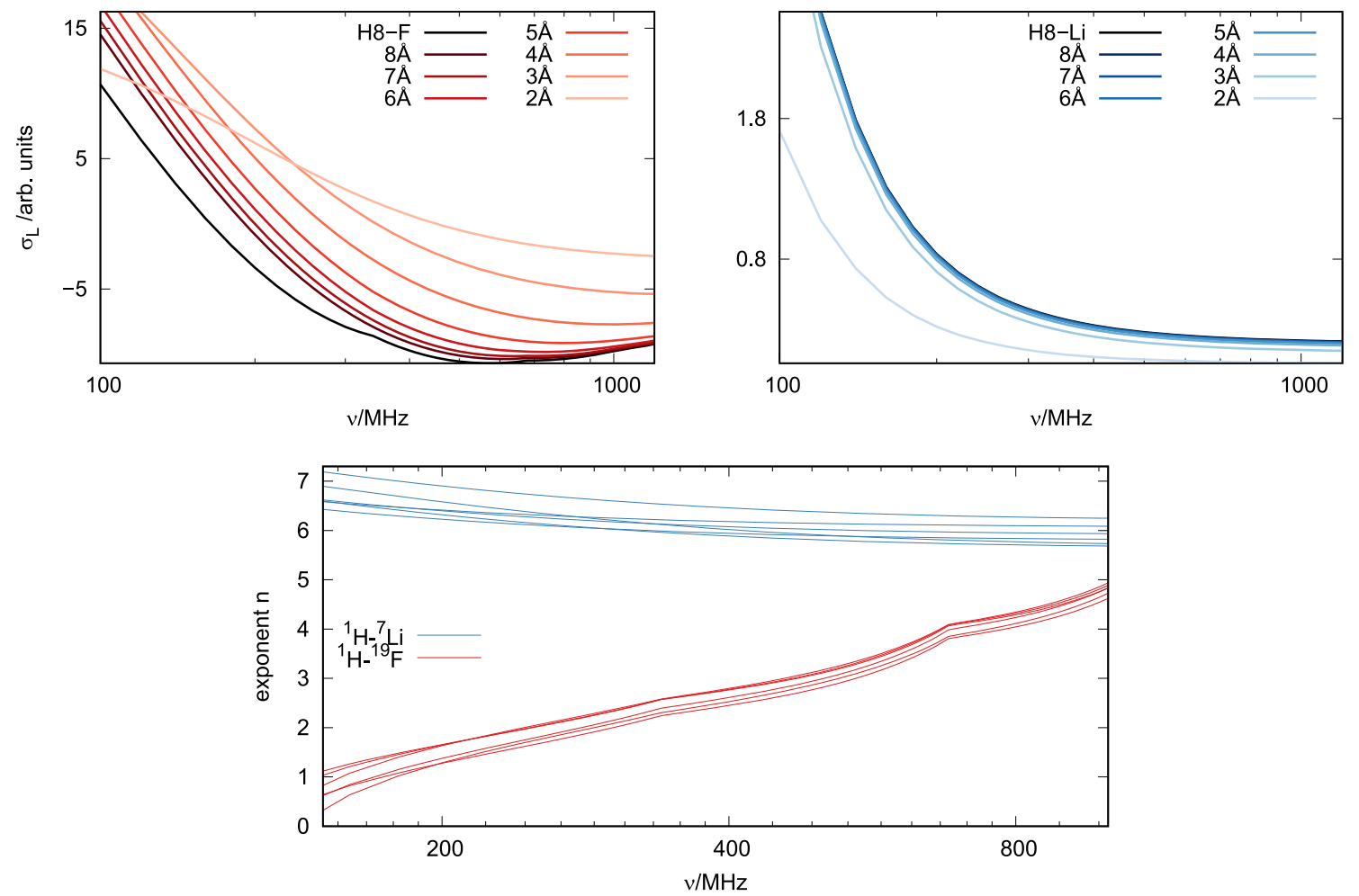

Figure 4. Cumulative contributions of shell-resolved cross-relaxation rates $\sigma_{\mathrm{L}}(r, \nu)$ of the $\mathrm{H} 8-\mathrm{F}$ spin pairs (left) and the $\mathrm{H} 8-\mathrm{Li}$ spin pairs (right). $\sigma_{\mathrm{L}}$ converges faster for ${ }^{1} \mathrm{H}-{ }^{7} \mathrm{Li}$ spin pairs than ${ }^{1} \mathrm{H}-{ }^{19} \mathrm{~F}$ spin pairs. Bottom: Interaction range of the $\mathrm{H}-\mathrm{F}$ spin pairs (red) and the $\mathrm{H}-\mathrm{Li}$ spin pairs (blue), represented by the exponent of the decay law $1 / r^{n}$.

length of at least $(100 \mathrm{MHz})^{-1}=10 \mathrm{~ns}$ (see Supporting Information for details).

We found cross-peaks of all $\mathrm{C}_{2} \mathrm{MIm}^{+}$protons interacting with the $\mathrm{CF}_{3}$ group of $\mathrm{OTf}^{-}$(Figure S3a) and $\mathrm{Li}^{+}$(Figure $\mathrm{S} 3 \mathrm{~b})$. The individual signal intensities are proportional to the magnitude of the given cross-relaxation and hint about the intensity of the mutual interaction. Figure 2 (top row) presents the NOEs of the neat IL $\left[\mathrm{C}_{2} \mathrm{MIm}\right][\mathrm{OTf}]$ and solution $0.9\left[\mathrm{C}_{2} \mathrm{MIm}\right] \cdot 0.1 \mathrm{Li} \cdot 1.0[\mathrm{OTf}]$. Some considerations can be drawn about the trends in experimental NOE values:

- The short-ranged ${ }^{1} \mathrm{H}-{ }^{7} \mathrm{Li}$ HOESY reflects specific $\mathrm{Li}$ cation interactions. Li preferentially interacts with $\mathrm{N}-$ $\mathrm{CH}_{3}$ (H8) and roughly equivalently with all other proton sites. The alkyl chain of imidazolium-based ILs is known to form hydrophobic domains, excluding charged functional groups. ${ }^{13,26}$ This can be seen in the ${ }^{1} \mathrm{H}-{ }^{7} \mathrm{Li}$ RDFs of the MD simulation as well (Figure S1): The $\mathrm{H} 8-\mathrm{Li}$ atom pair forms a higher peak at a short distance $(\approx 3-4 \AA)$ than either $\mathrm{H} 6-\mathrm{Li}$ and $\mathrm{H} 7-\mathrm{Li}$.

- ${ }^{1} \mathrm{H}-{ }^{19} \mathrm{~F}$ HOESY contains considerable contributions from the bulk. The most intense correlations of the anion are with the alkyl protons. Yet this behavior is neither chemically intuitive nor justified by the RDFs (Figure S1). The H-F NOEs are contaminated with unspecific long-ranged contributions and thus arbitrarily unreliable. In this respect, we observe that there is no considerable variation between the neat and Li-loaded samples.

For a semiquantitative analysis of HOESY cross-peaks, integrated volumes were corrected by a factor $N_{I} N_{S} /\left(N_{I}+\right.$ $N_{S}$ ), with $N_{I}$ the number of ${ }^{1} \mathrm{H}$ and $N_{S}$ the number of ${ }^{19} \mathrm{~F}$ or ${ }^{7} \mathrm{Li}$ nuclei contributing to the observed NOE signal. ${ }^{16,27-32}$
The corresponding corrected and normalized NOE build-up curves obtained from $29{ }^{1} \mathrm{H}-{ }^{19} \mathrm{~F}$ and $23{ }^{1} \mathrm{H}-{ }^{7} \mathrm{Li}$ spectra, at increasing mixing time, are displayed in Figures $3 \mathrm{a}$ and S4. As expected, from $20 \mathrm{~ms}$ to $700-900 \mathrm{~ms}$, a linear increase is observed in ${ }^{1} \mathrm{H}-{ }^{19} \mathrm{~F}$ build-up curves, then a maximum is reached, and an exponential decay is observed afterward. Similar behavior is seen in ${ }^{1} \mathrm{H}-{ }^{7} \mathrm{Li} \mathrm{HOESY}$, with the maximum shifted to $1.2 \mathrm{~s}$.

All curves were fitted using an exponential function derived from the fundamental Solomon equations, ${ }^{11,21}$ using $R$ (total longitudinal relaxation rate constant) and $\sigma_{I S}$ as fit-able parameters:

$$
\mathrm{NOE}=\frac{1}{2} \exp \left[-\left(R-\sigma_{I S}\right) \tau\right]\left(1-\exp \left[-2 \sigma_{I S} \tau\right]\right)
$$

Figure $3 \mathrm{~b}$ displays the cross relaxations $\sigma_{I S}$ obtained by fitting. Findings are in agreement with NOEs for both ${ }^{1} \mathrm{H}-{ }^{19} \mathrm{~F}$ and ${ }^{1} \mathrm{H}-{ }^{7} \mathrm{Li}$ interactions. As a result of the correction, the difference in intensity between the interactions at the different sites is reduced. For instance, looking at the ${ }^{1} \mathrm{H}-{ }^{19} \mathrm{~F}$ cross relaxations, those with the alkyl protons are still dominating, but the differences with the imidazolium protons are less significant. Similarly, the ${ }^{1} \mathrm{H}-{ }^{7} \mathrm{Li}$ cross-relaxation at $\mathrm{N}-\mathrm{CH}_{3}$ site has the highest value but less marked difference to the other protons.

The computational ${ }^{1} \mathrm{H}-{ }^{19} \mathrm{~F}$ and ${ }^{1} \mathrm{H}-{ }^{7} \mathrm{Li}$ NOEs are shown Figure 2 (second row). We find a reasonably good match between experimental and simulated NOEs (Figure 2, bottom). The most significant observed difference is in the ${ }^{1} \mathrm{H}-{ }^{7} \mathrm{Li} \mathrm{NOE}$ of the imidazolium $\mathrm{H} 4$ and $\mathrm{H} 5$ protons in the mixture and the ${ }^{1} \mathrm{H}-{ }^{19} \mathrm{~F}$ NOE of the $\mathrm{CH}_{2}$ protons in the neat IL. Since the overall trend of NOEs is faithfully reproduced, 
these four diverging values out of 33 spin pairs are not systematic and are likely local artifacts. The polarizabilities used in this work are more or less a function of the hybridization and the number of attached protons but take less into account the immediate chemical environment. Nevertheless, the emerging induced dipoles of these carbons based on these polarizabilities react individually to their local environment. Of course, an exact quantum-mechanical determination of the polarizabilities is also possible s3,34 $^{33}$ and leads to slight variations in the respective carbon polarizabilities and hence slightly different induced dipoles, but using new polarizabilities would require a complete reparametrization of the polarizable force field. The one applied in this work, however, has already proven to reproduce experimental NMR results. ${ }^{35}$

The reasonable agreement with the experimental values validates the accuracy of the $\mathrm{MD}$ simulation; thus the computational NOE calculations can be used to decompose observable sum spectra into different components. We dissect $\sigma_{\mathrm{L}}(\nu)$ into contributions from spin pairs at different distances,

$$
\sigma_{\mathrm{L}}(\nu)=\sum_{r=0}^{r_{\max }} \sigma_{\mathrm{L}}(r, \nu)
$$

Figures 4 (top) and S5 display the convergence to the experimentally observable $\sigma_{\mathrm{L}}(\nu)$.

The ${ }^{1} \mathrm{H}-{ }^{19} \mathrm{~F}$ contributions converge at larger distances than ${ }^{1} \mathrm{H}-{ }^{7} \mathrm{Li}$ contributions, meaning the latter are barely affected by spin interactions with the bulk. In addition, the frequency dependence of the ${ }^{1} \mathrm{H}-{ }^{19} \mathrm{~F}$ spin pairs is more pronounced: The spacing between the curve bundles varies from wide (low $\nu$, long-ranged) to small (high $\nu$, short-ranged). In order to quantify the changed range dependence, we fitted the spatially resolved $\sigma_{\mathrm{L}}(r, \nu)$ to a $1 / r^{n}$ law, shown in Figure 4(bottom). The $\mathrm{H}-\mathrm{Li}$ spin pairs show a relatively consistent short-ranged $1 / r^{6}$ distance dependence. The range of $\mathrm{H}-\mathrm{F}$ spin-pairs is long and additionally depends on the spectrometer frequency.

In summary, this contribution follows up the 2013 milestone paper by Gabl, Steinhauser, and Weingärtner, who introduced the fundamental concept that the structural information from intermolecular NOE is severely affected by the Larmor frequency of the interacting nuclei: "frequency does matter", 15 strongly discouraging the usage of intermolecular NOEs for structure determination in the chemist's community. Their work studied a ${ }^{1} \mathrm{H}-{ }^{19} \mathrm{~F}$ NOE.

Here we demonstrate how gyromagnetic ratios of interacting nuclei determine the intermolecular NOE range. The larger their difference, the larger the Larmor frequency difference $\mid \nu_{I}$ $-\nu_{S}$ becomes, avoiding the long-ranged low-frequency limit. We studied the IL electrolyte $0.9\left[\mathrm{C}_{2} \mathrm{MIm}\right] \cdot 0.1 \mathrm{Li} \cdot 1.0[\mathrm{OTf}]$ as a prototypical example with at least two remarkable outcomes:

- The good agreement between HOESY measurements and calculations validates the correctness of the computational results.

- Computational signal decomposition confirms that the ${ }^{1} \mathrm{H}-{ }^{19} \mathrm{~F}$ signal contains significant interactions with the bulk. In contrast, the ${ }^{1} \mathrm{H}-{ }^{7} \mathrm{Li}$ signal converges at small distances and is thus specific.

Our work provides experimentalists with a clear-cut interpretation tool for the structural use of intermolecular NOE: Proper selection of isotopes with differing gyromagnetic ratios overcomes the fundamental long-ranged limitation of intermolecular NOEs and provides intermolecular structural information.

Finally, the long-range interpretation of intermolecular NOEs should not prevent chemists from measuring them, as their interpretation adds value to other structural methods for assessing mesoscopic order, such as WAXS, SAXS, or SANS. As previously shown by some of us, the joint use of NOEs, scattering techniques, and $\mathrm{MD}$ simulations is a powerful investigating tool for nanostructured liquids. ${ }^{36}$

\section{ASSOCIATED CONTENT}

\section{SI Supporting Information}

The Supporting Information is available free of charge at https://pubs.acs.org/doi/10.1021/acs.jpclett.1c02253.

Theory and experimental and computational methods in detail (PDF)

\section{AUTHOR INFORMATION}

\section{Corresponding Authors}

Franca Castiglione - Department of Chemistry, Materials and Chemical Engineering "G. Natta", Politecnico di Milano, 20133 Milano, Italy; ○ orcid.org/0000-0003-2413-8808; Email: franca.castiglione@polimi.it

Christian Schröder - Department of Computational Biological Chemistry, University of Vienna, 1090 Vienna, Austria; 10 orcid.org/0000-0002-2167-5096;

Email: christian.schroeder@univie.ac.at

\section{Authors}

Philipp Honegger - Department of Systems Biology, Harvard Medical School, Boston, Massachusetts 02115, United States; Department of Computational Biological Chemistry, University of Vienna, 1090 Vienna, Austria; (1) orcid.org/ 0000-0001-8225-671X

Maria Enrica Di Pietro - Department of Chemistry, Materials and Chemical Engineering "G. Natta", Politecnico di Milano, 20133 Milano, Italy; 이이.org/0000-0002-2370-1948

Chiara Vaccarini - Department of Chemistry, Materials and Chemical Engineering "G. Natta”, Politecnico di Milano, 20133 Milano, Italy

Alea Quant - Department of Computational Biological Chemistry, University of Vienna, 1090 Vienna, Austria

Othmar Steinhauser - Department of Computational Biological Chemistry, University of Vienna, 1090 Vienna, Austria; ○ orcid.org/0000-0003-0839-6460

Andrea Mele - Department of Chemistry, Materials and Chemical Engineering "G. Natta", Politecnico di Milano, 20133 Milano, Italy; CNR-SCITEC Istituto di Scienze e Tecnologie Chimiche, 20133 Milano, Italy; o orcid.org/ 0000-0002-0351-0538

Complete contact information is available at: https://pubs.acs.org/10.1021/acs.jpclett.1c02253

\section{Author Contributions}

${ }^{\perp}$ P.H. and M.E.D.P. contributed equally.

\section{Notes}

The authors declare no competing financial interest.

\section{ACKNOWLEDGMENTS}

M.E.D.P. thanks Politecnico di Milano for her postdoctoral fellowship in the framework of the "MSCA EF Master Class 2018 " funding program. 


\section{REFERENCES}

(1) Welton, T. Ionic liquids: a brief history. Biophys. Rev. 2018, 10, 691-706.

(2) Canongia Lopes, J. N. A.; Pádua, A. A. H. Nanostructural Organization in Ionic Liquids. J. Phys. Chem. B 2006, 110, 33303335.

(3) Triolo, A.; Russina, O.; Bleif, H.-J.; Di Cola, E. Nanoscale Segregation in Room Temperature Ionic Liquids. J. Phys. Chem. B 2007, 111, 4641-4644.

(4) Judeinstein, P.; Zeghal, M.; Constantin, D.; Iojoiu, C.; Coasne, B. Interplay of Structure and Dynamics in Lithium/Ionic Liquid Electrolytes: Experiment and Molecular Simulation. J. Phys. Chem. B 2021, 125, 1618-1631.

(5) Berrod, Q.; Ferdeghini, F.; Judeinstein, P.; Genevaz, N.; Ramos, R.; Fournier, A.; Dijon, J.; Ollivier, J.; Rols, S.; Yu, D.; et al. Enhanced ionic liquid mobility induced by confinement in $1 \mathrm{D}$ CNT membranes. Nanoscale 2016, 8, 7845-7848.

(6) Castiglione, F.; Saielli, G.; Mauri, M.; Simonutti, R.; Mele, A. Xenon Dynamics in Ionic Liquids: A Combined NMR and MD Simulation Study. J. Phys. Chem. B 2020, 124, 6617-6627.

(7) Mantz, R. A.; Trulove, P. C.; Carlin, R. T.; Osteryoung, R. A. ROESY NMR of Basic Ambient-Temperature Chloroaluminate Ionic Liquids. Inorg. Chem. 1995, 34, 3846-3847.

(8) Mele, A.; Tran, C. D.; De Paoli Lacerda, S. H. The Structure of a Room-Temperature Ionic Liquid with and without Trace Amounts of Water: The Role of $\mathrm{C}-\mathrm{H} \cdots \mathrm{O}$ and $\mathrm{C}-\mathrm{H} \cdots \mathrm{F}$ Interactions in 1-n-Butyl-3Methylimidazolium Tetrafluoroborate. Angew. Chem., Int. Ed. 2003, 42, 4364-4366.

(9) Zhu, H.; O’Dell, L. A. Nuclear magnetic resonance characterisation of ionic liquids and organic ionic plastic crystals: common approaches and recent advances. Chem. Commun. 2021, 57, 5609.

(10) Damodaran, K. Recent NMR Studies of Ionic Liquids. Annu. Rep. NMR Spectrosc. 2016, 88, 215-244.

(11) Neuhaus, D.; Williamson, M. P. The Nuclear Overhauser Effect in Structural and Conformational Analysis; Wiley-VCH: Weinheim, Germany, 2000.

(12) Halle, B. Cross-relaxation between macromolecular and solvent spins: The role of long-range dipole couplings. J. Chem. Phys. 2003, $119,12372$.

(13) Braun, D.; Steinhauser, O. The intermolecular NOE is strongly influenced by dynamics. Phys. Chem. Chem. Phys. 2015, 17, 85098517.

(14) Frezzato, D.; Rastrelli, F.; Bagno, A. Nuclear Spin Relaxation Driven by Intermolecular Dipolar Interactions: The Role of SoluteSolvent Pair Correlations in the Modeling of Spectral Density Functions. J. Phys. Chem. B 2006, 110, 5676-5689.

(15) Gabl, S.; Steinhauser, O.; Weingärtner, H. From Short-Range to Long-Range Intermolecular NOEs in Ionic Liquids: Frequency Does Matter. Angew. Chem., Int. Ed. 2013, 52, 9242-9246.

(16) Castiglione, F.; Appetecchi, G. B.; Passerini, S.; Panzeri, W.; Indelicato, S.; Mele, A. Multiple points of view of heteronuclear NOE: Long range vs short range contacts in pyrrolidinium based ionic liquids in the presence of Li salts. J. Mol. Liq. 2015, 210, 215-222.

(17) Martin, P.-A.; Chen, F.; Forsyth, M.; Deschamps, M.; O’Dell, L. A. Correlating Intermolecular Cross-Relaxation Rates with Distances and Coordination Numbers in Ionic Liquids. J. Phys. Chem. Lett. 2018, 9, 7072-7078.

(18) Gyabeng, D.; Qiao, L.; Zhang, H.; Oteo, U.; Armand, M.; Forsyth, M.; Chen, F.; O'Dell, L. A. Anion-cation interactions in novel ionic liquids based on an asymmetric sulfonamide anion observed by NMR and MD simulations. J. Mol. Liq. 2021, 327, 114879.

(19) Martin, P.-A.; Salager, E.; Forsyth, M.; O’Dell, L. A.; Deschamps, M. On the measurement of intermolecular heteronuclear cross relaxation rates in ionic liquids. Phys. Chem. Chem. Phys. 2018, $20,13357$.

(20) Gyabeng, D.; Martin, P.-A.; Pal, U.; Deschamps, M.; Forsyth, M.; O'Dell, L. A. Investigating Intermolecular Interactions in a DMEBased Hybrid Ionic Liquid Electrolyte by HOESY NMR. Front. Chem. 2019, 7, 4.
(21) Solomon, I. Relaxation Processes in a System of Two Spins. Phys. Rev. 1955, 99, 559.

(22) Schröder, C. Comparing reduced partial charge models with polarizable simulations of ionic liquids. Phys. Chem. Chem. Phys. 2012, 14, 3089-3102.

(23) Hwang, L.-P.; Freed, J. H. Dynamic effects of pair correlation functions on spin relaxation by translational diffusion in liquids. $J$. Chem. Phys. 1975, 63, 4017-4025.

(24) Ayant, Y.; Belorizky, E.; Fries, P.; Rosset, J. Intermolecular contribution to dipole-dipole relaxation rates of small molecules. J. Phys. (Paris) 1977, 38, 325-337.

(25) Bloembergen, N.; Purcell, E. M.; Pound, R. V. Relaxation effects in nuclear magnetic resonance absorption. Phys. Rev. 1948, 73, 679.

(26) Hayes, R.; Warr, G. G.; Atkin, R. Structure and Nanostructure in Ionic Liquids. Chem. Rev. 2015, 115, 6357.

(27) Hou, S.-S.; Tzeng, J.-K.; Chuang, M.-H. Intermolecular association and supramolecular structures of PNVF-LiPFN and PVP-LiPFN complexes in the aqueous phase. Soft Matter 2010, 6, 409-415.

(28) Khatun, S.; Castner, E. W., Jr Ionic liquid-solute interactions studied by 2D NOE NMR spectroscopy. J. Phys. Chem. B 2015, 119, 9225-9235.

(29) Castiglione, F.; Moreno, M.; Raos, G.; Famulari, A.; Mele, A.; Appetecchi, G. B.; Passerini, S. Structural organization and transport properties of novel pyrrolidinium-based ionic liquids with perfluoroalkyl sulfonylimide anions. J. Phys. Chem. B 2009, 113, 1075010759.

(30) Lee, H. Y.; Shirota, H.; Castner, E. W., Jr Differences in ion interactions for isoelectronic ionic liquid homologs. J. Phys. J. Phys. Chem. Lett. 2013, 4, 1477-1483.

(31) Zuccaccia, C.; Bellachioma, G.; Cardaci, G.; Macchioni, A. Solution structure investigation of $\mathrm{Ru}$ (II) complex ion pairs: Quantitative NOE measurements and determination of average interionic distances. J. Am. Chem. Soc. 2001, 123, 11020-11028.

(32) Di Pietro, M. E.; Castiglione, F.; Mele, A. Anions as Dynamic Probes for Ionic Liquid Mixtures. J. Phys. Chem. B 2020, 124, 28792891.

(33) Heid, E.; Szabadi, A.; Schröder, C. Quantum mechanical determination of atomic polarizabilities of ionic liquids. Phys. Chem. Chem. Phys. 2018, 20, 10992-10996.

(34) Bedrov, D.; Piquemal, J.-P.; Borodin, O.; MacKerell, A.; Roux, B.; Schröder, C. Molecular Dynamics Simulations of Ionic Liquids and Electrolytes Using Polarizable Force Fields. Chem. Rev. 2019, 119, $7940-7995$.

(35) Honegger, P.; Overbeck, V.; Strate, A.; Appelhagen, A.; Sappl, M.; Heid, E.; Schröder, C.; Ludwig, R.; Steinhauser, O. Understanding the Nature of Nuclear Magnetic Resonance Relaxation by Means of Fast-Field-Cycling Relaxometry and Molecular Dynamics Simulations-The Validity of Relaxation Models. J. Phys. Chem. Lett. 2020, 11, 2165.

(36) Lo Celso, F.; Yoshida, Y.; Castiglione, F.; Ferro, M.; Mele, A.; Jafta, C.; Triolo, A.; Russina, O. Direct experimental observation of mesoscopic fluorous domains in fluorinated room temperature ionic liquids. Phys. Chem. Chem. Phys. 2017, 19, 13101-13110. 